\title{
Large and sensitive magnetostriction in ferromagnetic composites with nanodispersive precipitates
}

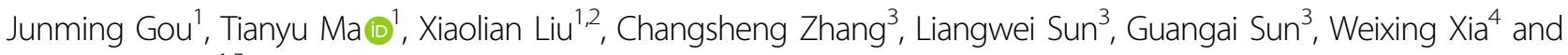
Xiaobing Ren (1) ${ }^{1,5}$

\begin{abstract}
Large and sensitive magnetostriction (large strain induced by small magnetic fields) is highly desired for applications of magnetostrictive materials. However, it is difficult to simultaneously improve magnetostriction and reduce the switching field because magnetostriction and the switching field are both proportional to the magnetocrystalline anisotropy. To solve this fundamental challenge, we report that introducing tetragonal nanoprecipitates into a cubic matrix can facilitate large and sensitive magnetostriction even in random polycrystals. As exhibited in a proof-ofprinciple reference, Fe-Ga alloys, the figure of merit-defined by the saturation magnetostriction over the magnetocrystalline anisotropy constant - can be enhanced by over 5-fold through optimum aging of the solutiontreated precursor. On the one hand, the aging-induced nanodispersive face-centered tetragonal (FCT) precipitates create local tetragonal distortion of the body-centered cubic (BCC) matrix, substantially enhancing the saturation magnetostriction to be comparable to that of single crystal materials. On the other hand, these precipitates randomly couple with the matrix at the nanoscale, resulting in the collapse of net magnetocrystalline anisotropy. Our findings not only provide a simple and feasible approach to enhance the magnetostriction performance of random polycrystalline ferromagnets but also provide important insights toward understanding the mechanism of heterogeneous magnetostriction.
\end{abstract}

\section{Introduction}

Magnetostrictive materials that can convert magnetic energy into mechanical energy or vice versa have been widely used in actuators, transducers, sensors, and energy harvesters ${ }^{1-3}$. For most applications, it is desirable to have a combination of large strain and a small switching field (which can be evaluated by the critical field, at which $d_{33}$ reaches a maximum ${ }^{4}$, and is proportional to $K / M_{\mathrm{s}}$ for

\footnotetext{
Correspondence: Tianyu Ma (matianyu@xjtu.edu.cn) or Xiaobing Ren (ren. xiaobing@nims.go.jp)

${ }^{1}$ Frontier Institute of Science and Technology, State Key Laboratory for Mechanical Behavior of Materials, and MOE Key Laboratory for Nonequilibrium Synthesis and Modulation of Condensed Matter, Xi'an Jiaotong University, Xi'an 710049, China

${ }^{2}$ School of Materials and Environmental Engineering, Hangzhou Dianzi University, Hangzhou 310012, China

Full list of author information is available at the end of the article
}

random polycrystals, where $K$ is the magnetocrystalline anisotropy constant and $M_{\mathrm{s}}$ is the saturation magnetiza$\operatorname{tion}^{5}$ ). However, it is difficult to simultaneously obtain a large magnetostriction and small switching field in a homogeneous ferromagnet because both the magnetostriction and switching fields are proportional to the magnetocrystalline anisotropy (MCA $)^{1}$. There is one exception: combining two terminals with large magnetostriction but opposite MCA constants can yield large magnetostriction with reduced switching fields at a specific composition/ temperature, as found in the well-known pseudobinary intermetallic compounds $R R^{\prime} \mathrm{Fe}_{2}$ ( $R$ and $R^{\prime}$ refer to two rare earth elements $)^{2,6-8}$. When maximizing the magnetostriction for a given material, there is still a trade-off between a large magnetostriction and a small switching field, analogous to the strength-ductility trade-off in structural

\section{(c) The Author(s) 2021}

(c) (i) Open Access This article is licensed under a Creative Commons Attribution 4.0 International License, which permits use, sharing, adaptation, distribution and reproduction cc) in any medium or format, as long as you give appropriate credit to the original author(s) and the source, provide a link to the Creative Commons license, and indicate if changes were made. The images or other third party material in this article are included in the article's Creative Commons license, unless indicated otherwise in a credit line to the material. If material is not included in the article's Creative Commons license and your intended use is not permitted by statutory regulation or exceeds the permitted use, you will need to obtain permission directly from the copyright holder. To view a copy of this license, visit http://creativecommons.org/licenses/by/4.0/. 
materials. The usual approach to maximize the magnetostriction of a given material with a large spontaneous magnetostriction constant has been fabricating a single crystal and further applying prestress or performing postgrowth magnetic annealing ${ }^{9-11}$. This complex process can constrain the ferromagnetic domains within the plane perpendicular to the crystal axis and can yield a large magnetostriction up to the theoretical limit through pure $90^{\circ}$ domain switches when applying a magnetic field along the crystal axis. For decades, this approach has dominated the fabrication and application of the state-of-the-art magnetostrictive material Terfenol-D (e.g., $\mathrm{Tb}_{0.3} \mathrm{Dy}_{0.7} \mathrm{Fe}_{2}$ ) with a large rhombohedral spontaneous magnetostriction constant $\lambda_{111}$ of $1640 \mathrm{ppm}$ at room temperature ${ }^{2}$. The obtained technical strain $\lambda_{/ / \mathrm{s}}$ along the field direction can be as high as $2400 \mathrm{ppm}$ (nearly equivalent to the theoretical limit $3 / 2 \lambda_{111}$ ) by applying compressive prestress on the single crystal ${ }^{11,12}$. However, a large magnetostriction is always obtained at the expense of an enlarged switching field (i.e., deteriorated sensitivity) because prestress and magnetic annealing inevitably induce additional anisotropy. Consequently, it is highly desirable to find a feasible approach that can enhance the magnetostriction and reduce the switching field simultaneously.

Several theoretical studies separately note that the exchange of nonequal crystallographic axes of the tetragonal strain nanodomains embedded within a cubic matrix will output large magnetostriction ${ }^{13}$, and the random magnetic coupling between them will result in the collapse of net $\mathrm{MCA}^{14,15}$. Experimentally, it is feasible to obtain such a nanostructured composite by aging a cubic supersaturated solid solution to form nanodispersive tetragonal precipitates, as extensively reported in structural alloys. In this work, we obtained ferromagnetic composites by isothermal aging of the metastable cubic precursor to precipitate nanodispersive tetragonal particles. Our results reveal that this can be a simple and feasible approach to obtain the desired large and sensitive magnetostriction in random polycrystals, as exhibited by the proof-of-principle reference Fe-Ga alloys.

The selection of $\mathrm{Fe}-\mathrm{Ga}$ alloys takes into account the large magnetostriction at small fields and, in particular, the apparent structural differences between metastable and equilibrium states. In fact, $\mathrm{Fe}-\mathrm{Ga}$ alloys have stimulated extensive research efforts since their large magnetostriction was discovered in $2000^{16}$. As shown in two review papers ${ }^{17,18}$, previous research focused mainly on the compositions in the vicinity of 19 and $27 \mathrm{Ga}$ at.\%, at which two magnetostriction peaks have been reported ${ }^{19}$. The obtained $\lambda_{/ / s}$ of these two alloys has been successfully enhanced from $\sim 60 \mathrm{ppm}$ for random polycrystals ${ }^{16}$ to $\sim 200 \mathrm{ppm}$ for [001]-oriented single crystals or polycrystals $^{20,21}$ and further to above $300 \mathrm{ppm}$ (near the theoretical limit $3 / 2 \lambda_{100}$ ) by applying a coaxial compressive prestress or by performing postgrowth magnetic/stress annealing ${ }^{9,10,21,22}$. More importantly, these two alloys bear an average body-centered cubic (BCC) structure in the quenched state but have biphase $\mathrm{A} 2+\mathrm{L}_{2}$ (ordered facecentered cubic) for $\mathrm{Fe}_{81} \mathrm{Ga}_{19}$ and single $\mathrm{L} 1_{2}$ for $\mathrm{Fe}_{73} \mathrm{Ga}_{27}$ in the equilibrium state (see the phase diagram in Fig. S1) ${ }^{23}$. Since the $\mathrm{L}_{2}$ phase has negative magnetostriction (in contrast to the $\mathrm{A} 2, \mathrm{~B} 2$ and $\mathrm{D}_{3}$ phases) ${ }^{24-27}$, approaching equilibrium conditions has been undesirable for achieving large positive magnetostriction. However, as shown hereinafter, short-term aging of the $\mathrm{BCC}$ precursor induces $\mathrm{L6}_{0}$-type face-centered tetragonal (FCT) nanoprecipitates instead of the equilibrium $\mathrm{L}_{2}$ phase. The unique nanostructure with nanodispersive FCT precipitates embedded in the BCC matrix developed by optimum aging can create fivefold enhancement in the figure of merit due to the simultaneous magnetostriction enhancement and the collapse of net MCA. The obtained $\lambda_{/ / \mathrm{s}}$ in the optimally aged $\mathrm{Fe}_{81} \mathrm{Ga}_{19}$ random polycrystal can reach as high as $200 \mathrm{ppm}$, which is comparable to that for single crystal materials measured under stress-free conditions $^{20,21}$.

\section{Materials and methods}

Ingots with nominal compositions of $\mathrm{Fe}_{81} \mathrm{Ga}_{19}$ and $\mathrm{Fe}_{73} \mathrm{Ga}_{27}$ were prepared by induction melting $\mathrm{Fe}$ and $\mathrm{Ga}$ with purities of $99.99 \%$ in an argon atmosphere. Cylindrical rods were prepared by subsequent casting into a steel mold with an inner diameter of $12 \mathrm{~mm}$. The rods were sealed in quartz tubes filled with high-purity argon and subjected to heat treatment. The metastable BCC precursors were obtained by annealing the rods for $72 \mathrm{~h}$ at $1373 \mathrm{~K}$, followed by quenching in ice water. An electron back-scattered diffraction (EBSD, HITACHI-SU3500) image of the $1373 \mathrm{~K}$-quenched sample revealed randomly oriented grains of several hundred microns (Fig. S2). The $\mathrm{Fe}_{73} \mathrm{Ga}_{27}$ and $\mathrm{Fe}_{81} \mathrm{Ga}_{19}$ rods were then cut into different sections $\left(\varnothing 12 \times 20 \mathrm{~mm}^{3}\right)$ and aged at $723 \mathrm{~K}$ or $673 \mathrm{~K}$, followed by quenching in ice water.

Note that during aging, the $\mathrm{Fe}-\mathrm{Ga}$ alloys undergo a diffusion-controlled phase transformation ${ }^{25-27}$, after which the microstructure has a strong surface effect. To avoid the surface effect, sheets with dimensions of $10 \times$ $3 \times 1.5 \mathrm{~mm}^{3}$ were cut from the center sections of the aged rods (the length direction was parallel to the rod axis) and subjected to the following magnetostriction measurements and microstructural characterizations. Room temperature initial magnetization curves were measured using a SQUID magnetometer, which allows us to calculate the MCA constant $K_{1}$ and saturation magnetization $M_{\mathrm{s}}$ according to the law of approaching saturation. Magnetostriction along the magnetic field direction was measured at room temperature using the strain gauge method. The strain gauge had a length of $1 \mathrm{~mm}$ and a 
width of $1.5 \mathrm{~mm}$ (KYOWA, KFR-1-120-C1-11), which was much larger than the grain size of several hundred microns for the solution-treated sample (Fig. S2) and several microns for the overaged samples. To avoid possible stress-induced impurities, the sample surface was carefully polished by standard mechanical grinding and electropolishing methods. At least five magnetization cycles (one cycle refers to increasing the magnetic field from zero to a designed strength, decreasing it to zero, then increasing the opposite magnetic field to the same designed strength, and finally decreasing it to zero) were measured for each sample. In addition, a random polycrystalline $\mathrm{Fe}_{72} \mathrm{Ga}_{28}$ alloy was selected as another reference to reproduce the aging effect on magnetostriction properties. Nanoindentation (G200) and atomic force microscopy (AFM) were conducted on the polished surfaces to investigate the modulus difference between the nanoprecipitates and the matrix. The foils for TEM characterization were prepared by twin-jet electropolishing at temperatures below $243 \mathrm{~K}$. TEM characterizations were performed using a JEOL JEM-2100F microscope at $200 \mathrm{kV}$. The high-resolution TEM (HRTEM) images and geometric phase analysis (GPA) were analyzed using DigitalMicrograph (Gatan). Magnetic domain structures were observed under the Fresnel Lorentz mode. An inplane magnetic field was applied to observe the domain structure evolution. The optimally aged $\mathrm{Fe}_{73} \mathrm{G}_{27}$ sample (cut into sheets with dimensions of $10 \times 10 \times 1.5 \mathrm{~mm}^{3}$ ) was subjected to in situ small-angle neutron scattering (SANS) measurements using unpolarized neutrons with a wavelength of $5.3 \AA$, which was performed on the smallangle neutron spectrometer at the China Mianyang Research Reactor (CMRR). An in-plane magnetic field was applied along the length direction.

\section{Results and discussion}

\section{Aging-time-dependent magnetostriction and microstructure}

Figures 1 and 2 show the magnetostriction and magnetization curves of the $\mathrm{Fe}_{73} \mathrm{Ga}_{27}$ and $\mathrm{Fe}_{81} \mathrm{Ga}_{19}$ samples, respectively. Both alloys exhibit similar aging-timedependent magnetostrictions. In the solution-treated state, the saturation magnetostriction along the field direction $\left(\lambda_{/ / \mathrm{s}}\right)$ for both alloys is $50-65 \mathrm{ppm}$ (varies with composition), which is comparable to the values for $\mathrm{Fe}-\mathrm{Ga}$ random polycrystals reported by Clark et al. ${ }^{16}$. After isothermal aging, the magnetostriction first increases to a peak value and then decreases to nearly zero or negative values with increasing aging time. Figure 1a shows the aging-time-dependent $\lambda_{/ / \mathrm{s}}$ for $\mathrm{Fe}_{73} \mathrm{Ga}_{27}$ random polycrystals, where $\lambda_{/ / \mathrm{s}}$ increases significantly from $65 \mathrm{ppm}$ for the $1373 \mathrm{~K}$-quenched state to $172 \mathrm{ppm}$ for the one aged for $1 \mathrm{~h}$ at $723 \mathrm{~K}$ but gradually decreases to $-75 \mathrm{ppm}$ for the one aged for $12 \mathrm{~h}$. Representative

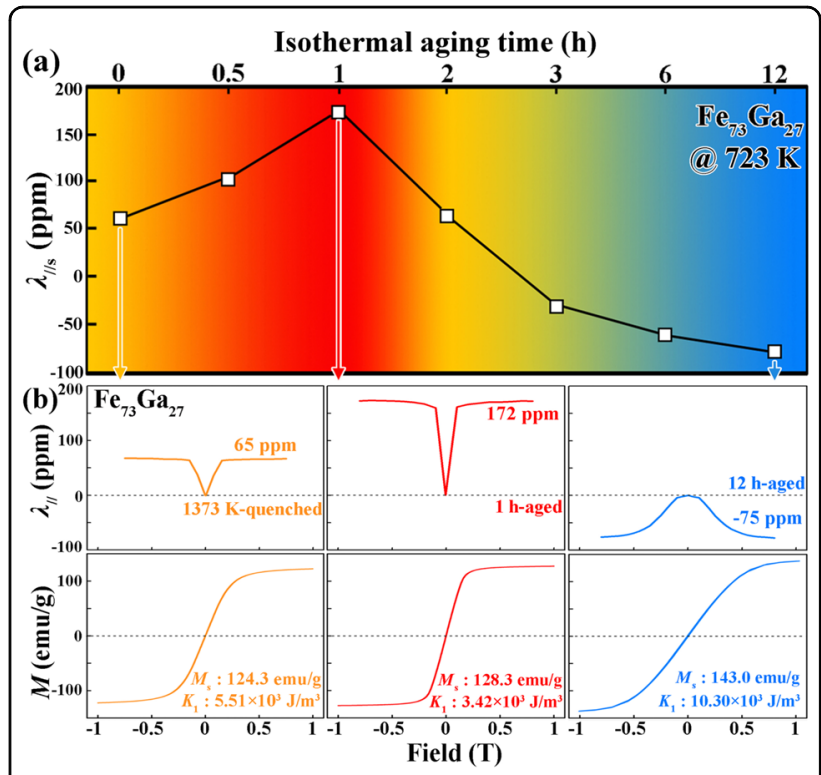

Fig. 1 Magnetostriction and magnetization of $\mathrm{Fe}_{73} \mathrm{Ga}_{27}$. a Agingtime-dependent saturation magnetostriction for $\mathrm{Fe}_{73} \mathrm{Ga}_{27}$ random polycrystals. b Selected magnetostriction, initial magnetization curves, and magnetization hysteresis loops.

magnetostriction curves are shown in Fig. 1b. The highest magnetostriction $\lambda_{/ / \mathrm{s}}$ for the $\mathrm{Fe}_{81} \mathrm{Ga}_{19}$ alloy is $202 \mathrm{ppm}$ after aging for $8 \mathrm{~h}$ at $673 \mathrm{~K}, \sim 4$ times that for the solutiontreated state (Fig. 2). Further magnetostriction measurements on the $\mathrm{Fe}_{72} \mathrm{Ga}_{28}$ random polycrystalline samples reproduce this aging time dependence (Fig. S3). More importantly, the obtained $202 \mathrm{ppm}$ magnetostriction is comparable to that for a single crystal measured under stress-free conditions ${ }^{20,21}$ and surpasses the reported values in bulk $\mathrm{Fe}-\mathrm{Ga}$ random polycrystals doped with a third element, such as $\mathrm{Cr}, \mathrm{Mn}, \mathrm{Co}$, Dy, or $\mathrm{Tb}^{19,28-31}$, as shown in Fig. 2d. Therefore, short-term isothermal aging is a simple and feasible approach to achieve large magnetostriction in $\mathrm{Fe}-\mathrm{Ga}$ random polycrystals without doping with any expensive and scarce metals or the need to grow single crystals on a special instrument.

Isothermal aging also results in the collapse of the net MCA, thereby significantly enhancing the figure of merit, as shown in Fig. 2c. The MCA constant $K_{1}$ calculated according to the law of approaching saturation exhibits a nonlinear dependence on aging time. $K_{1}$ for the $1 \mathrm{~h}$-aged $\mathrm{Fe}_{73} \mathrm{Ga}_{27}$ sample and the $8 \mathrm{~h}$-aged $\mathrm{Fe}_{81} \mathrm{Ga}_{19}$ sample is even smaller than that for the $1373 \mathrm{~K}$-quenched sample, which is unusual for a homogeneous ferromagnet since a larger magnetostriction should result from strengthened $\mathrm{MCA}^{1}$. The reduced $K_{1}$ (collapse of net MCA) indicates that the alloys become much softer after optimum aging. The figure of merit can be defined by $\left.\left|\lambda_{/ / \mathrm{s}} / H_{\mathrm{s}}\right|\right|^{32}$ or $\left|\lambda_{/ / \mathrm{s}}\right|$ $\left.K_{1}\right|^{33}$ or $\left|\lambda_{/ / \mathrm{s}} / H_{\mathrm{c}}\right|^{7}$, where $H_{\mathrm{s}}$ is the magnetostriction saturation field and $H_{\mathrm{c}}$ is the coercivity. Since both $H_{\mathrm{s}}$ and 

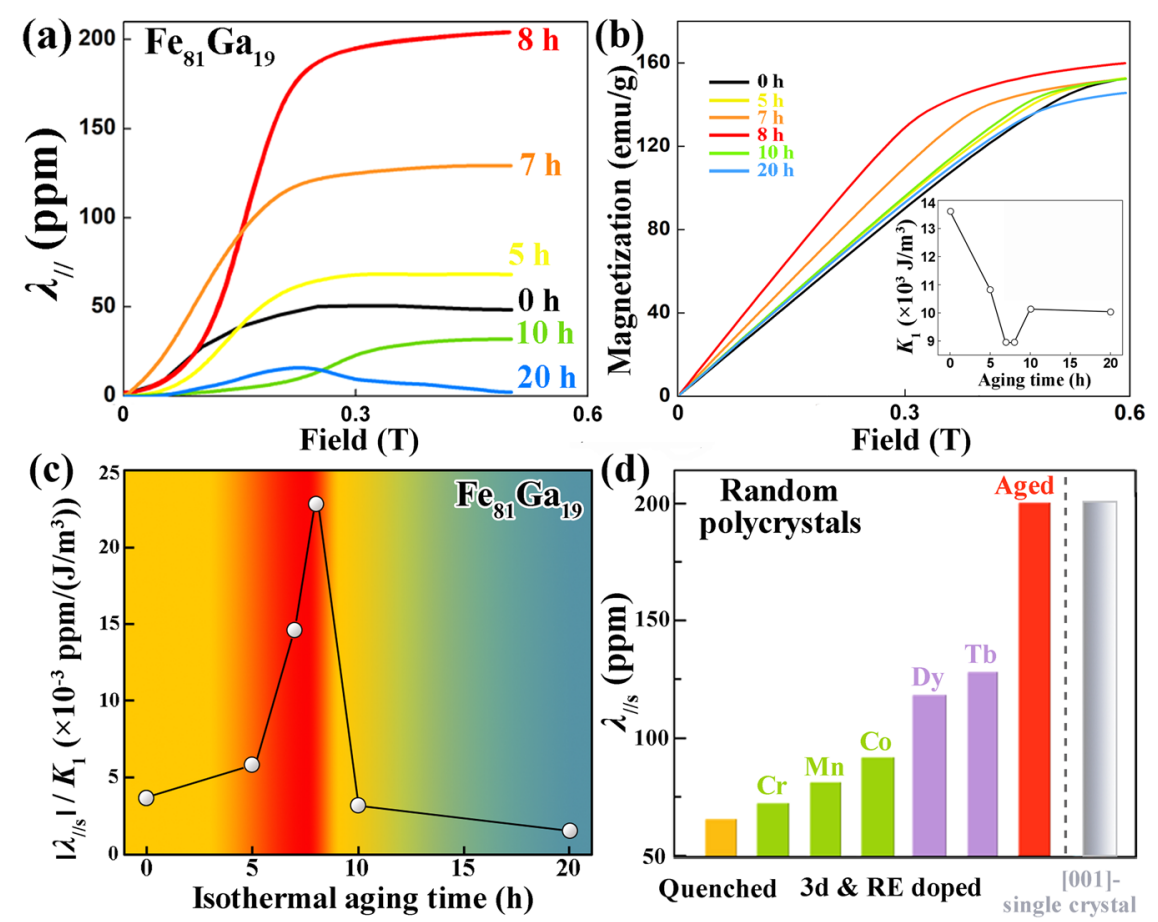

Fig. 2 Magnetostriction and magnetization of $\mathrm{Fe}_{\mathbf{8 1}} \mathrm{Ga}_{\mathbf{1 9}}$. $\mathbf{a}$, $\mathbf{b}$ Magnetostriction curves and initial magnetization curves for $\mathrm{Fe}_{81} \mathrm{Ga}_{19}$ with different heat treatments. The inset in $\mathbf{b}$ is the aging time dependence of the magnetocrystalline anisotropy constant $K_{1}$. $\mathbf{c}$ Figure of merit of magnetostriction as a function of aging time for the $\mathrm{Fe}_{81} \mathrm{Ga}_{19}$ random polycrystals. $\mathbf{d}$ Comparison of $\lambda_{/ / 5}$ among our optimally aged Fe-Ga random polycrystals, the single crystals (measured under stress-free conditions ${ }^{20,21}$ ) and the quenched random polycrystals doped with a third element in refs. ${ }^{19,28-31}$.

$H_{\mathrm{c}}$ are proportional to $\left|K_{1}\right|$ for random polycrystalline ferromagnets, here, we use $\left|\lambda_{/ / s} / K_{1}\right|$ to evaluate the figure of merit of the Fe-Ga random polycrystals. The $\left|\lambda_{/ / \mathrm{s}} / K_{1}\right|$ for the optimally aged $\mathrm{Fe}_{81} \mathrm{Ga}_{19}$ sample is nearly 5 times larger than that of the quenched state, as shown in Fig. 2c. This means that after optimum aging, the magnetostriction becomes more sensitive, i.e., it is very easy to achieve large and sensitive magnetostriction. In addition, the $1 \mathrm{~h}$ aged $\mathrm{Fe}_{73} \mathrm{Ga}_{27}$ sample exhibits only 3.2\% higher saturation magnetization $M_{\mathrm{s}}$ than the $1373 \mathrm{~K}$-quenched sample (Fig. 1b) but $186.7 \%$ larger $\lambda_{/ / \mathrm{s}}$. This is also against conventional wisdom for homogeneous ferromagnets because $\left|\lambda_{\mathrm{s}}\right|$ is proportional to $M_{\mathrm{s}}^{31}$.

Figure 3 shows bright-field images and the corresponding selected area electron diffraction (SAED) patterns of the $\mathrm{Fe}_{73} \mathrm{Ga}_{27}$ samples with different heat treatments. The superlattice reflections at the $\{200\}^{*}$ positions suggest that the $\mathrm{BCC}$ phase is ordered at the asquenched state (Fig. 3d). According to previous stu$\operatorname{dies}^{26,34}$, this state contains a relatively large fraction of the $\mathrm{D}_{3}$ phase in addition to the disordered A2 phase. After aging for $1 \mathrm{~h}$ at $723 \mathrm{~K}$, randomly distributed nanoprecipitates are formed (Fig. 3b). Most nanoprecipitates have a similar size of $\sim 20 \mathrm{~nm}$, whereas few have a size of $\sim 80 \mathrm{~nm}$. These nanoprecipitates produce additional superlattice reflections around the $\{110\}^{*}-\mathrm{D} 0_{3}$ positions that are excluded for $\mathrm{A} 2, \mathrm{~B} 2$ or $\mathrm{D}_{3}$, as indicated by the red arrows in Fig. 3e. After aging for $12 \mathrm{~h}$ at $723 \mathrm{~K}$, the nanoprecipitates transform into $\mathrm{L}_{2}$ with a mean grain size of $\sim 1 \mu \mathrm{m}$ (Fig. 3c, f).

Figure 4a shows a typical HRTEM image taken along the [001] zone axis (ZA) of the $1 \mathrm{~h}$-aged $\mathrm{Fe}_{73} \mathrm{Ga}_{27}$ sample. Region I has the $\mathrm{D}_{3}$ structure. However, the FFT pattern of region II displays additional superlattice reflections around the $\{110\}^{*}-\mathrm{DO}_{3}$ positions, as indicated by the red arrows. These extra superlattice reflections can be indexed as (010), (100), (010) and (100) spots for an FCT L6 6 -type structure, according to the Bain orientation relationship [001]-FCT//[001]-D0 3 and (200)-FCT// (220)-D0 $0_{3}$. Along [111] ZA, the FCT nanoprecipitates produce additional superlattice reflections around the $(\overline{1} 10)-\mathrm{DO}_{3}$ and $(\overline{11} 2)-\mathrm{D0}_{3}$ positions (Fig. $4 \mathrm{~d}$ ), which can be indexed as (010)-FCT and (101)-FCT, following the Bain orientation relationship [111]- $\mathrm{D0}_{3} / /[101]-\mathrm{FCT}$ and (202)-D0 $/ /(\overline{1} 11)-F C T$. Along [110] ZA, the FCT nanoprecipitates produce additional superlattice reflections around the $(\overline{1} 10)-\mathrm{DO}_{3}$ and $(\overline{1} 12)-\mathrm{DO}_{3}$ positions (Fig. $\left.4 \mathrm{~g}\right)$, which can be indexed as (010)-FCT and (011)-FCT, following the Bain orientation relationship [110]- $\mathrm{D0}_{3} / /[100]$ FCT and $(\overline{2} 20)-\mathrm{DO}_{3} / /(020)-\mathrm{FCT}$. It should be noted that 


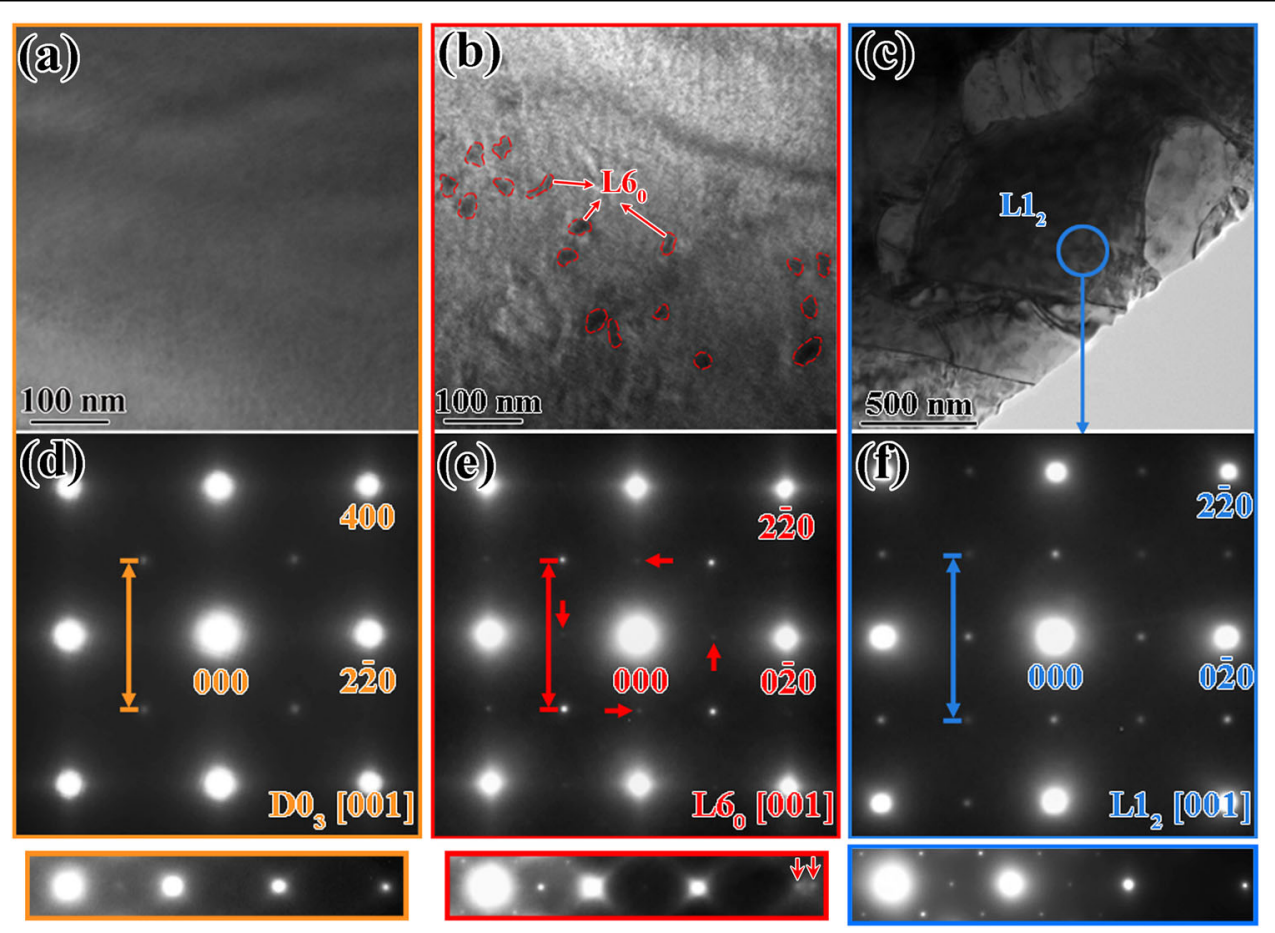

Fig. 3 TEM characterizations of $\mathrm{Fe}_{\mathbf{7 3}} \mathrm{Ga}_{\mathbf{2 7}}$. Bright-field images and SAED patterns for the $\mathbf{a}, \mathbf{d} 1373 \mathrm{~K}$-quenched, $\mathbf{b}$, e $1 \mathrm{~h}$-aged and $\mathbf{c}$, $\mathbf{f} 12 \mathrm{~h}$-aged $\mathrm{Fe}_{73} \mathrm{Ga}_{27}$ random polycrystals. Enlarged electron diffraction patterns for each state are illustrated at the bottom.

the $\mathrm{L6}_{0}$-type FCT structure is alternatively a bodycentered-tetragonal (BCT)-modified $\mathrm{DO}_{3}$ structure, as suggested by Lograsso et al. ${ }^{35}$. The obtained FFT patterns are consistent with the simulated patterns for the modified $\mathrm{DO}_{3}$ structure, as reported by Jiang et al. ${ }^{36-38}$. $\mathrm{L6}_{0^{-}}$ type nanoprecipitates are also observed in the $8 \mathrm{~h}$-aged $\mathrm{Fe}_{81} \mathrm{Ga}_{19}$ alloy (Fig. S4).

An important conclusion can then be drawn: the shortterm aging-induced substantial magnetostriction enhancement is attributed to the formation of $L 6_{0}$-type nanoprecipitates, and the overaging-induced magnetostriction deterioration is due to the further transformation into the $\mathrm{L} 1_{2}$ phase with intrinsic negative magnetostriction. It should be noted that, in the past, the quenched state has been believed to exhibit the largest magnetostriction, and approaching the equilibrium state (e.g., slow cooling or increasing aging time) will deteriorate the magnetostriction ${ }^{39-41}$. Numerous investigations have shown that slow cooling will promote the ordering of the $\mathrm{DO}_{3}$ phase and/or the precipitation of the $\mathrm{L}_{2} / \mathrm{DO}_{19}$ phases, which are detrimental to magnetostriction performance $^{34,42-44}$. For instance, A.K. Mohamed et al. recently showed that the $\mathrm{A} 2 \rightarrow \mathrm{D0}_{3}+\mathrm{A} 2^{\prime}$ transition will occur above $673 \mathrm{~K}$ with cooling rates of $2-30 \mathrm{~K} / \mathrm{min}$ and that the $\mathrm{L}_{2}$ phase will appear with cooling rates $\leq 2 \mathrm{~K} /$ min in $\mathrm{Fe}_{80.5} \mathrm{Ga}_{19.5}$ alloys ${ }^{43}$. On the other hand, long-term aging within the $\mathrm{L1}_{2}$ phase regime will lead to a large fraction of the $\mathrm{L}_{2}$ phase and deteriorate magnetostriction $^{24,27,45}$. For instance, the magnetostriction of the $\mathrm{Fe}_{72.4} \mathrm{Ga}_{27.6}$ alloy decreases from 105 to $30 \mathrm{ppm}$ when the 50 vol.\% $\mathrm{L}_{2}$ phase is formed ${ }^{27}$. However, the present work reveals that placing $\mathrm{Fe}-\mathrm{Ga}$ alloys at a proper intermediate state between the metastable and equilibrium states (e.g., short-term aging) should be the optimum condition to maximize magnetostriction, owing to the formation of dispersive $\mathrm{LG}_{0}$ nanoprecipitates. The role of $\mathrm{L} 6_{0}$ nanoprecipitates in enhancing magnetostriction will be discussed in the next section.

\section{Role of FCT nanoprecipitates}

Note that the formation of the $\mathrm{L}_{2}$ phase requires simultaneous atomic migration (by aligning Ga-Ga pairs along the [001] direction) and drastic lattice distortion (simultaneous elongation along the [001] axis by $25.9 \%$ and contractions along the [100] and [010] axes by $-11.1 \%$ ) of the metastable BCC phases ${ }^{26,46}$; thus, it has slow kinetics. Therefore, the transformation process and the product phase are largely affected by the competition between the strain energy and interfacial energy. In the early transformation stage, given that the atomic positions within the nanoprecipitates have been altered to fit the $\mathrm{L}_{2}$ lattice, the lattice distortion will be constrained by the surrounding matrix because of the limited size. As shown in Fig. 3, the extra superlattice reflections of the 

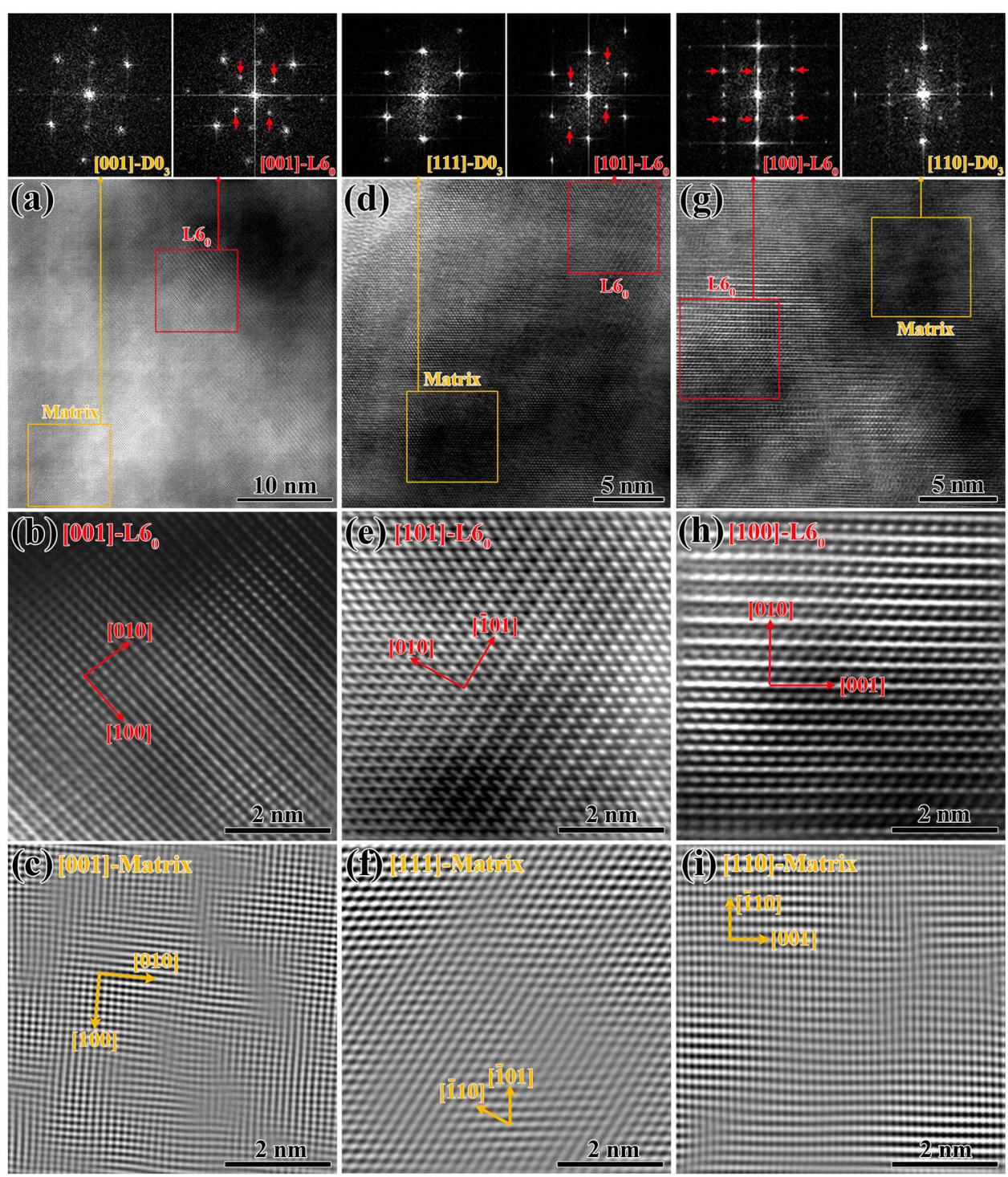

Fig. 4 HRTEM characterizations of the $1 \mathbf{h}$-aged $\mathrm{Fe}_{\mathbf{7 3}} \mathrm{Ga}_{\mathbf{2 7}}$. HRTEM image, selected area FFT patterns, and IFFT images of the $1 \mathrm{~h}$-aged $\mathrm{Fe}_{73} \mathrm{Ga}_{27}$ sample along the a-c [001], d-f [111], and $\mathbf{g}-\mathbf{i}$ [110] ZAs. The IFFT images in (c), (f) and (i) were taken from the matrix using the (040), (040), (400),

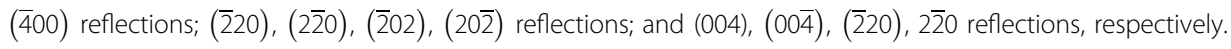

L6 0 nanoprecipitates indicate that the atomic position exchanges occur at local regions; however, the lattice parameters are not fixed for the equilibrium $\mathrm{L}_{2}$. For instance, the calculated tetragonality $(c / a)$ for the FCT L6 0 nanoprecipitates is 0.712 in Fig. 4e, h, which is slightly greater than $1 / \sqrt{2}$ for an ideal BCC structure and much smaller than 1 for an ideal FCC structure. In this case, the $\mathrm{L}_{0}$ nanoprecipitates can be deemed the constrained- $\mathrm{L} 1_{2}$ phase. The unit cells of $\mathrm{DO}_{3}, \mathrm{~L}_{0}$ and $\mathrm{L1}_{2} \mathrm{Fe}_{3} \mathrm{Ga}$ are shown in Fig. S5. Very recently, our work indicated that L6 0 nanoprecipitates have size-dependent tetragonality; the larger their size is, the greater the tetragonality (closer to 1 of the equilibrium $\mathrm{L1}_{2}$ phase) ${ }^{47}$.
It should be noted that the BCC matrix has an open structure and that the $\mathrm{L}_{0}$ and $\mathrm{L} 1_{2}$ phases are more closely packed. The nanoindentation test revealed that the $1 \mathrm{~h}$ aged sample yields the precipitation hardening effect due to the formation of $\mathrm{L}_{0}$ nanoprecipitates, which are mechanically harder than the $\mathrm{BCC}$ matrix (Fig. S6). The mutual elastic interaction between the harder $\mathrm{L}_{0}$ nanoprecipitates and the softer $\mathrm{BCC}$ phase locally distorts the surrounding matrix, although the formation of $\mathrm{L} 6_{0}$ multivariants reduces the net strain. One can see from the diffraction pattern in Fig. 3e that diffuse scattering of the fundamental reflections indicates local lattice distortion. More clearly, the lattice distortion is reflected by the 
splitting feature along the [100]* direction. As indicated by the red arrows in the bottom of Fig. 3e, the high-order fundamental reflection $(1200)-\mathrm{DO}_{3}$ in the $1 \mathrm{~h}$-aged $\mathrm{Fe}_{73} \mathrm{Ga}_{27}$ sample splits into two weaker reflections. In contrast, the $1373 \mathrm{~K}$-quenched and $12 \mathrm{~h}$-aged samples do not have such apparent splitting features, as shown in the bottoms of Fig. 3d, f, respectively. Figure 4c shows a typical distorted region surrounding the $\mathrm{L}_{0}$ nanoprecipitate, which is an inverse FFT image created by taking

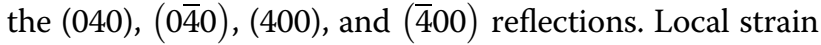
fluctuations can be seen from the inequivalent $d$ spacings and between the (010) and (100) planes. Such lattice distortion is also observed along the [111]- and [110]- $\mathrm{D0}_{3}$ zone axes, as shown in Fig. 4f, i, respectively. The local lattice distortion surrounding the nanoprecipitates is also verified by geometric phase analysis (Fig. S7). The local tetragonal distortion then gives rise to an apparent average tetragonality greater than 1 [splitting of the (1200)$\mathrm{DO}_{3}$ reflection in the bottom of Fig. 3e], which means that the spontaneous magnetostriction constant $\lambda_{001}$ of the matrix is effectively increased after short-term aging [compared with the (1200)-D0 ${ }_{3}$ reflection without splitting in the bottom of Fig. 3d].

Figure $5 \mathrm{a}-\mathrm{f}$ show the in-, over- and under-focus L-TEM Fresnel images of the $1373 \mathrm{~K}$-quenched and $1 \mathrm{~h}$-aged $\mathrm{Fe}_{73} \mathrm{Ga}_{27}$ samples, respectively. The bright and black lines are the $90^{\circ}$ and $180^{\circ}$ magnetic domain walls (DWs). The domain of closure is observed for the $1373 \mathrm{~K}$-quenched sample. Interestingly, after aging for $1 \mathrm{~h}$, magnetic DWs are observed at the phase interfaces (marked by the red arrows in Fig. 5e). Such DWs are due to the domain wall energy density difference between the matrix and $\mathrm{L}_{0}$ nanoprecipitates. Unlike the straight and well-ordered samples for the $1373 \mathrm{~K}$-quenched sample, after aging, the DWs transform into serpentine and irregularly shaped configurations. This indicates that the $\mathrm{L}_{0}$ nanoprecipitates are magnetically coupled with the matrix phase. The irregular magnetic domain configurations of the matrix are the result of the collapse of net anisotropy, which stems from the competition between the coherent anisotropy and the random anisotropy of the $\mathrm{L}_{0}$ nanoprecipitates $^{15}$. Figure $5 \mathrm{~g}-\mathrm{i}$ show in situ L-TEM images for the $1 \mathrm{~h}$-aged $\mathrm{Fe}_{73} \mathrm{Ga}_{27}$ sample. When a small magnetic field of $0.006 \mathrm{~T}(60 \mathrm{Oe})$ is applied, the magnetic DWs of some L6 0 nanoprecipitates disappear and the ferromagnetic domains of the matrix simultaneously rotate toward the field direction. When the magnetic field increases to $0.015 \mathrm{~T}$ (150 Oe), the DWs of the matrix disappear, whereas those of several $\mathrm{L}_{0}$ nanoprecipitates still exist. This indicates that the $L 6_{0}$ nanoprecipitates with easy axes far away from the field direction are certainly more difficult to magnetize than those with easy axes close to the field direction.

We further performed in situ SANS measurements on the $1 \mathrm{~h}$-aged $\mathrm{Fe}_{73} \mathrm{Ga}_{27}$ sample. Figure $6 \mathrm{a}-\mathrm{d}$ show the original 2D SANS patterns detected in different magnetic fields. The contour of 2D patterns undergoes an isotropyanisotropy-isotropy transition with increasing magnetic field. Figure $6 \mathrm{e}, \mathrm{f}$ show the scattering intensity $I(q)$ curves summed over the scattering vectors $q_{\mathrm{x}}$ (parallel to $H$ ) and $q_{\mathrm{y}}$ (perpendicular to $H$ ) from 2D patterns, respectively. In the $I-q_{\mathrm{x}}$ curves (Fig. 6e), a broad peak is detected in the range of $0.1 \mathrm{~nm}^{-1} \leq q_{\mathrm{x}} \leq 0.3 \mathrm{~nm}^{-1}$ at $0 \mathrm{~T}, 0.05 \mathrm{~T}$ and $0.15 \mathrm{~T}$; however, this peak disappears when the field increases to $0.5 \mathrm{~T}$ or $1.7 \mathrm{~T}$. In the $I-q_{\mathrm{y}}$ curves (Fig. $6 \mathrm{f}$ ), a broad peak is also observed at $0 \mathrm{~T}$ and $0.05 \mathrm{~T}$, whereas this peak disappears at fields above $0.15 \mathrm{~T}$. This magneticfield-dependent broad peak corresponds to inhomogeneous $\mathrm{L}_{0}$ nanoprecipitates. In the nanoprecipitate-matrix approximation ${ }^{48,49}$, the scattering intensity $I(q)$ reflects the spatial fluctuations of scattering length density (SLD) for both nuclear and magnetic contributions. The nuclear contribution can be expressed as $N V_{p}^{2}|F(q)|^{2} \Delta \rho_{\text {nuc }}^{2}$, and the magnetic contribution can be expressed as $N V_{p}^{2}|F(q)|^{2} \Delta \rho_{\text {mag }}^{2} \sin ^{2} \alpha$, where $N$ stands for the number of nanoprecipitates; $V_{p}$ is the volume of an individual nanoprecipitate with the form factor $F(q) ; \Delta \rho_{\text {nuc }}$ and $\Delta \rho_{\text {mag }}$ are the contrasts of nuclear and magnetic SLDs between the nanoprecipitates and the matrix, respectively; and $\alpha$ is the angle between $q$ and the local magnetization vector in the nanoprecipitates.

The calculated nuclear SLD is $\sim 7.2 \times 10^{-6} \AA^{-2}$ for both the $\mathrm{BCC}$ matrix and the $\mathrm{L}_{0}$ nanoprecipitates (Fig. S8), which is consistent with the value reported in the literature $^{50}$. This indicates that there is no measurable nuclear contrast between the $\mathrm{L}_{0}$ nanoprecipitate and matrix. In addition, since the $\mathrm{L}_{0}$ nanoprecipitates formed by diffusive phase transformation cannot be decomposed by increasing the magnetic field, the magnetic-fielddependent broad peak is not attributed to their nuclear contrast but rather to the magnetic contrast between the $\mathrm{L6}_{0}$ nanoprecipitates and the matrix. Under $1.7 \mathrm{~T}$ (at which the magnetization is saturated), all the magnetizations are parallel to the applied field direction, and the $I-q_{\mathrm{y}}$ curve can then reflect the magnetic contribution due to the contrast of magnetic SLDs between the $\mathrm{L}_{0}$ nanoprecipitates and matrix. According to the fitting curve (Fig. S8a), the calculated average radius for the spherical nanoprecipitates is $9.7 \pm 0.1 \mathrm{~nm}$ (consistent with the TEM observations) and the calculated $\Delta \rho_{\text {mag }}$ (the difference in magnetic SLDs between the $\mathrm{L6}_{0}$ nanoprecipitates and matrix) is $\sim 4.0 \times 10^{-7} \AA$. We further calculated the magnetic SLD for the BCC matrix and the $\mathrm{L}_{0}$ nanoprecipitates, which is $2.9 \times 10^{-6} \AA^{-2}$ and $3.3 \times 10^{-6} \AA^{-2}$, respectively.

The distinct feature between the $I-q_{\mathrm{x}}$ and $I-q_{\mathrm{y}}$ curves at $0.15 \mathrm{~T}$ can correlate the magnetization flips of the $\mathrm{L} 6_{0}$ nanoprecipitates with the magnetostriction since the magnetostriction is nearly saturated in this field (Fig. 1b). 

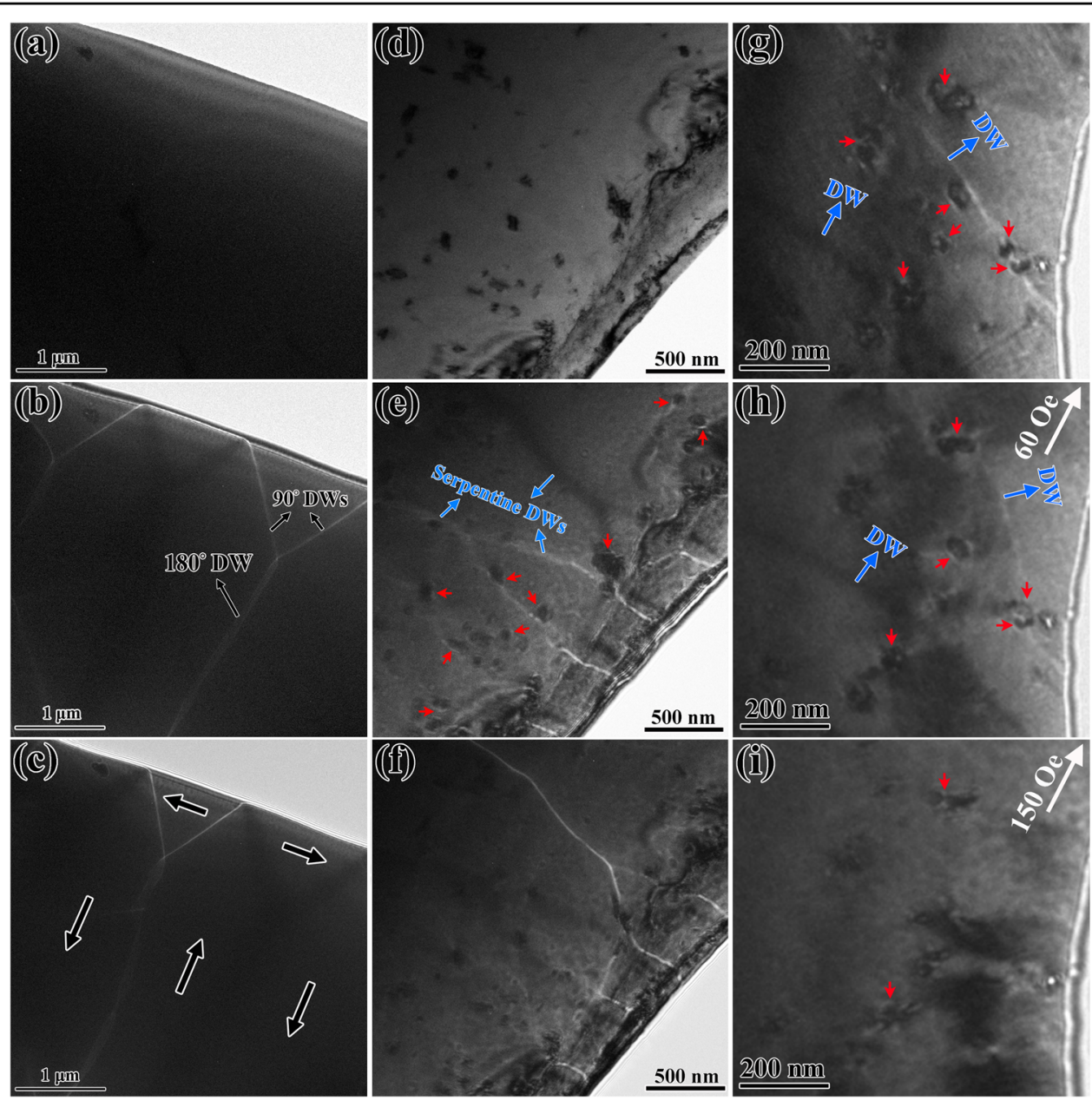

Fig. 5 Magnetic domain structure and evolution for $\mathrm{Fe}_{73} \mathrm{Ga}_{27}$. In-, over- and under-focus Lorentz-TEM images for the a-c solution-treated and d-f 1 h-aged $\mathrm{Fe}_{73} \mathrm{Ga}_{27}$ samples. $\mathbf{g}$-i In situ over-focus Lorentz-TEM images for the $1 \mathrm{~h}$-aged $\mathrm{Fe}_{73} \mathrm{Ga}_{27}$ sample taken at 0 T, 0.006 T (60 Oe) and 0.015 T $(150$ Oe). The white arrow indicates the in-plane field direction.

The magnetization process of the $1 \mathrm{~h}$-aged $\mathrm{Fe}_{73} \mathrm{Ga}_{27}$ sample can be derived from the in situ characterizations, as schematically illustrated in Fig. $6 \mathrm{~g}-\mathrm{i}$. For ease of understanding, the nanoprecipitates with the $c$-axis parallel (or close) and perpendicular to (or far away from) $H$ are defined as $\mathrm{L} 6_{0 / /}$ and $\mathrm{L} 6_{0 \perp}$, respectively. According to the observations of the DWs surrounding the $\mathrm{L}_{0}$ nanoprecipitates in Fig. 5e, g, the directions of spontaneous magnetizations for the tetragonal $\mathrm{L}_{0}$ nanoprecipitates are distinct from the surrounding $\mathrm{BCC}$ matrix phase at the thermally demagnetized state $(0 \mathrm{~T})$. Determined from the factor $\sin ^{2} \alpha$ in the form $N V_{p}^{2}|F(q)|^{2} \Delta \rho_{\text {mag }}^{2} \sin ^{2} \alpha$, the peak in the $I-q_{\mathrm{x}}$ curve arises from the magnetic contrast between the magnetizations of the $\mathrm{L} 6_{0 \perp}$ nanoprecipitates and the vertical components of the matrix magnetizations. The peak in the $I-q_{\mathrm{y}}$ curve then arises from the magnetic contrast between the magnetizations of the $L 6_{0 / /}$ nanoprecipitates and the parallel components of the matrix magnetizations. According to the literature ${ }^{51,52}$, the different magnetization directions of the nanoprecipitates and matrix can engender strong magnetic scattering. When the field increases to $0.15 \mathrm{~T}$ (Fig. 6h), the magnetizations in the $\mathrm{L}_{0 / /}$ nanoprecipitates flip toward $H$ together with the rotation of the ferromagnetic domains of the matrix. This results in magnetic contrast collapse in the $I-q_{\mathrm{y}}$ curve, i.e., the disappearance of the peak at $0.15 \mathrm{~T}$. Moreover, the magnetizations of the $\mathrm{L}_{0 \perp}$ nanoprecipitates remain far from $H$ since the peak still exists in the $I-q_{\mathrm{x}}$ curve, indicating that $0.15 \mathrm{~T}$ is not sufficiently strong to switch the magnetizations of the $\mathrm{L}_{0 \perp}$ nanoprecipitates along the field direction, which is consistent with the L-TEM observation in Fig. 5i, where the DWs of the matrix disappear and those of several $\mathrm{L}_{0}$ nanoprecipitates still exist. Following the literature ${ }^{48,49}$, the field dependence of the aspect ratio of scattering intensity $\left[I\left(q_{\mathrm{y}}\right) / I\left(q_{\mathrm{x}}\right)\right]$ summed within the peak range $\left(0.1 \mathrm{~nm}^{-1}<q\right.$ $<0.3 \mathrm{~nm}^{-1}$ ) is plotted in Fig. S8b. Since the magnetizations of the $\mathrm{L}_{0 / /}$ and $\mathrm{L}_{0 \perp}$ nanoprecipitates may 

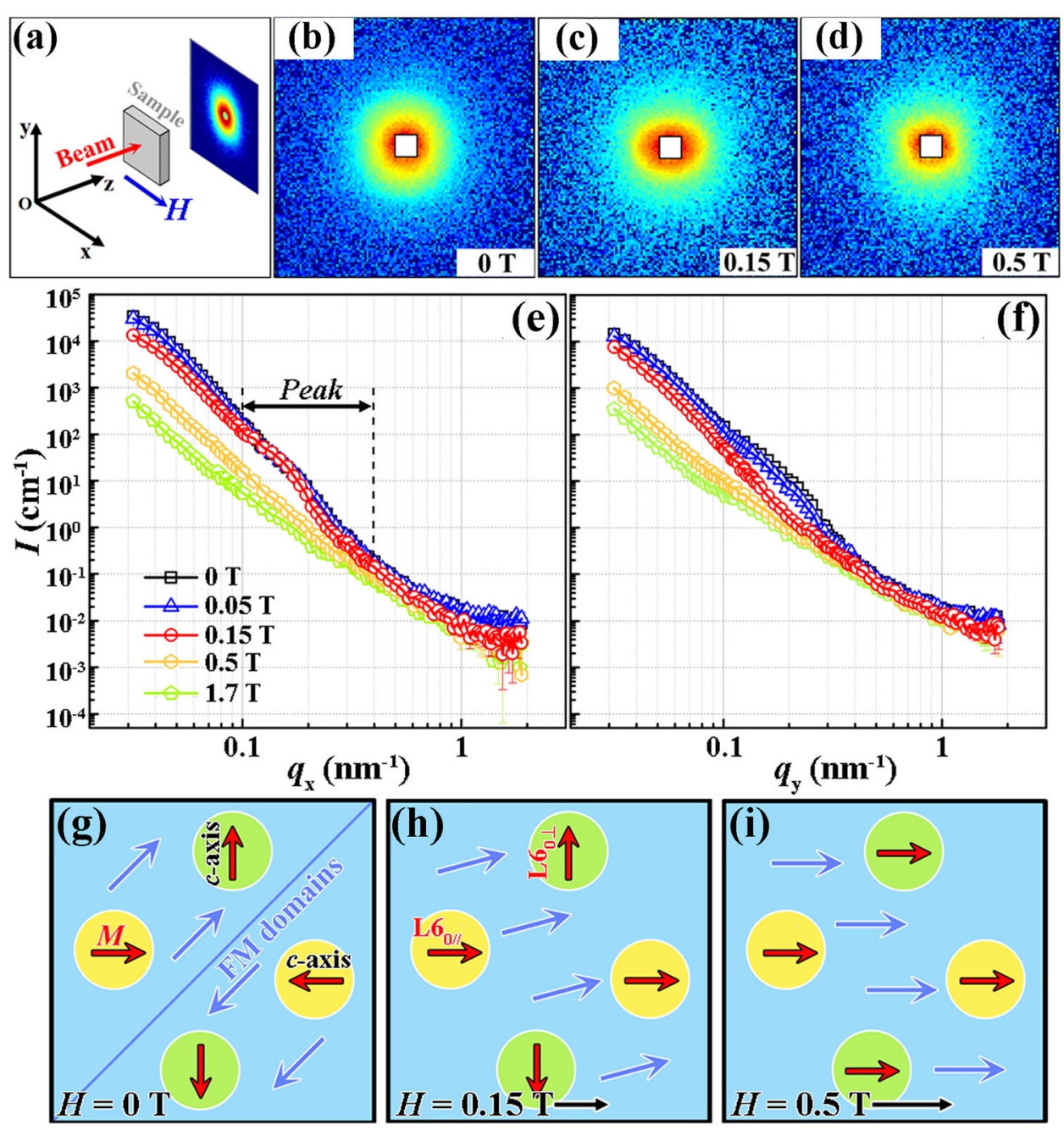

Fig. $\mathbf{6}$ In situ SANS characterization of the $\mathbf{1}$ h-aged $\mathrm{Fe}_{\mathbf{7 3}} \mathrm{Ga}_{\mathbf{2 7}}$ sample. a Schematic of SANS measurements and in situ 2D scattering patterns obtained at $\mathbf{b} 0, \mathbf{c} 0.15$, and $\mathbf{d} 0.5 \mathrm{~T}$. e, f $l-q_{\mathrm{x}}$ and $l-q_{\mathrm{y}}$ curves obtained at $0,0.05,0.15,0.5$, and $1.7 \mathrm{~T}$. g-i Schematic magnetization flips at different magnetic fields. The abbreviated "FM" refers to ferromagnetic.

simultaneously rotate with the ferromagnetic domains of the matrix, the aspect ratio will increase with increases in the magnetic field owing to the increased magnetic scattering in the direction perpendicular to the field. However, the aspect ratio gradually decreases to $\sim 0.36$ as the field increases to $0.15 \mathrm{~T}$, which confirms the nonuniform flipping of the magnetizations of the $\mathrm{L} 6_{0 / /}$ and $\mathrm{L}_{0 \perp}$ nanoprecipitates. The increased magnetic contrast between the magnetizations of the $L 6_{0 \perp}$ nanoprecipitates and the vertical components of the matrix magnetizations gives rise to the relatively enhanced scattering intensity for $I-q_{\mathrm{x}}$, and the reduced magnetic contrast between the magnetizations of the L6// nanoprecipitates and the parallel components of the matrix magnetizations gives rise to the relatively decreased scattering intensity for $I-q_{y}$. When the field further increases to $0.5 \mathrm{~T}$ (Fig. 6i), the magnetizations of the $\mathrm{L} 6_{0 \perp}$ nanoprecipitates are also nearly oriented along the field direction, as determined from the magnetic contrast collapse in the $I-q_{\mathrm{x}}$ curve and the further increase in the aspect ratio (Fig. S8b). Such results indicate that the large magnetostriction generated below $0.15 \mathrm{~T}$ arises mainly from the magnetization flips of the ferromagnetic domains of the matrix and the L6// nanoprecipitates. Considering that the magnetization flips of the $\mathrm{L}_{0 \perp}$ nanoprecipitates should result in larger magnetostriction than those of the $\mathrm{L}_{0 / /}$ ones, the SANS measurements then exclude the contribution of the magnetization flips of the $\mathrm{L}_{0 \perp}$ nanoprecipitates to the large magnetostriction obtained in the present Fe-Ga alloys.

\section{Discussion}

The present findings may have important implications. 
First, this work may provide important insights toward understanding the microstructure origin of the large magnetostriction in Fe-Ga alloys. In fact, it is still an open question why soluting nonmagnetic $\mathrm{Ga}$ into $\mathrm{Fe}$ can induce a significant magnetostriction enhancement. In particular, intrinsic factors, namely, Ga-induced changes in the electronic structures of the A2 solid solution, were proposed to explain this phenomenon ${ }^{53-55}$. Note that $\mathrm{Fe}-\mathrm{Ga}$ alloys are inherently heterogeneous at the nanoscale. Both macroscopic and local techniques have identified that in addition to the A2 matrix ${ }^{26,34-38,56,57}$, quenched Fe-Ga alloys also contain short-range-ordered $\mathrm{Ga}-\mathrm{Ga}$ pairs $\left(<2 \mathrm{~nm}\right.$ ), forming cubic $\mathrm{D0}_{3}$ nanoprecipitates with $\mathrm{Ga}-\mathrm{Ga}$ pairs along the $\langle 110\rangle$ axis of the A2 matrix and/or tetragonal $\mathrm{L} 6_{0}$-type nanoprecipitates with $\mathrm{Ga}-\mathrm{Ga}$ pairs along the $\left\langle 001>_{\mathrm{BCC}}\right.$ axis. It is clear that the cubic $\mathrm{D}_{3}$ nanoprecipitates are detrimental since slow cooling promotes the long-range-ordering of the $\mathrm{DO}_{3}$ phase and deteriorates magnetostriction $^{39-42}$. It was shown that proper heat treatment can avoid the formation of $\mathrm{DO}_{3}$ nanoprecipitates, thereby effectively enhancing the magnetostriction of Fe-Ga alloys. For instance, $\lambda_{/ / \mathrm{s}}$ of the $\mathrm{Fe}_{81} \mathrm{Ga}_{19}$ alloy can be enhanced by $71.4 \%$ when quenched near the phase boundary between $\mathrm{A} 2$ and $\left(\mathrm{A} 2+\mathrm{L}_{2}\right)$ after slow cooling (free of $\mathrm{DO}_{3}$ nanoprecipitates), in comparison with the $\mathrm{D0}_{3}$-bearing sample quenched at high temperature $(1273 \mathrm{~K})^{58}$. Comparably, the present approach of shortterm aging can provide a more significant magnetostriction enhancement (e.g., magnetostriction increased 3-fold after aging the $\mathrm{Fe}_{81} \mathrm{Ga}_{19}$ alloy for $8 \mathrm{~h}$ ), given that harmful $\mathrm{DO}_{3}$ ordering occurs simultaneously with the formation of L6 $6_{0}$ nanoprecipitates. This indicates that the $\mathrm{L}_{0}$-type nanoprecipitates should play an important role in enhancing magnetostriction in addition to the intrinsic factors. The present work contributes to finding a feasible approach to enlarge the size and enrich the population of L6 0 -type nanoprecipitates that strongly affect the magnetostriction of $\mathrm{Fe}-\mathrm{Ga}$ alloys, thus giving rise to substantial magnetostriction enhancement. In addition, our work also helps to understand why L6 $6_{0}$-type nanoprecipitates form in Fe-Ga alloys. During rapid cooling, the high vacancy density leads to the short-range-ordering of Ga-Ga pairs along the $<001>$ direction, forming very fine $\mathrm{L}_{0}$ nanoprecipitates. When subjected to further aging, the fine $\mathbf{L} 6_{0}$ nanoprecipitates grow toward forming the $\mathrm{L}_{2}$ equilibrium phase, which requires simultaneous atomic position changes and lattice distortion of the matrix. In the early transformation stage, the $\mathrm{L}_{2}$ precursors are constrained at the nanoscale by the untransformed matrix. As the aging time extends, equilibrium $\mathrm{L}_{2}$ particles are preferably formed in local regions. Because of its intrinsic negative magnetostriction, it will offset the positive magnetostriction of the distorted matrix, leading to a decrease in net magnetostriction, as shown in Fig. 1. Consequently, the optimum condition to obtain large magnetostriction in Fe-Ga alloys is to form L6 $6_{0}$ nanoprecipitates in the intermediate state. It should also be noted that this diffusion-controlled phase transformation is highly sensitive to the material dimensions since the atomic diffusion rate is much faster at the material surface than at the internal material surface. When utilizing this strategy to obtain large magnetostriction in Fe-Ga alloys, the optimum aging time for a fixed composition depends on the sample size, for instance, the thickness of sheet samples. As shown in Fig. S9, after aging for $1 \mathrm{~h}$ at $723 \mathrm{~K}$, the surface of a $1 \mathrm{~mm}$ thick $\mathrm{Fe}_{73} \mathrm{Ga}_{27}$ sheet sample already contained mainly the $\mathrm{L}_{2}$ phase.

Second, this work provides important insights into the heterogeneous magnetostriction mechanism of ferromagnetic composites with nanoscale precipitation. Early investigations have suggested that the matrix surrounding the nanoprecipitates rather than the nanoprecipitates themselves is the source of the large magnetostriction of $\mathrm{Fe}-\mathrm{Ga}$ alloys $\mathrm{s}^{36,37,49,56}$. Within the tetragonal nanoprecipitate model proposed by Khachaturyan et al. ${ }^{59}$, the tetragonal nanoprecipitates rotate by realigning the $c$-axis or distort in concert with the much larger ferromagnetic domains, and elastic coupling of nanoprecipitates to the matrix results in large magnetostriction. Jiang et al. ${ }^{36}$ suggested that the nanoprecipitates already generated tetragonal distortion of the cubic matrix and that rotation of the matrix tetragonality results in large magnetostriction. Our work supports the local tetragonal distortion of the matrix. The distorted matrix shares a general morphology with premartensitic tweeds or unfrozen strain glass $^{60-62}$, which contain a very fine mixture of variously oriented spontaneous strain states at the nanoscale. According to the model proposed by Seiner et al. ${ }^{61}$, reorientation of the premartensitic tweeds in Ni-Mn-Ga alloy can be easily induced by a small magnetic field with a magnetostriction of $\sim 200 \mathrm{ppm}$. Similar low-field-triggered large magnetostriction has also recently been reported in ferromagnetic $\mathrm{Fe}-\mathrm{Pd}$ strain glass with tetragonal strain nanodomains embedded in a cubic matrix ${ }^{32}$. The presently aged $\mathrm{Fe}-\mathrm{Ga}$ alloys with local tetragonal distortion are new members of this family. In addition, our in situ SANS investigations suggested that the magnetization flips of the L6 $6_{0}$ nanoprecipitates (those with magnetizations perpendicular to the field direction) occur at high fields, thereby excluding their direct role in the large magnetostriction at low fields. A recent phase field simulation work ${ }^{37}$ is consistent with this experimental finding, in which the $\mathrm{Ga}-\mathrm{Ga}$ pairs (along the [001] easy axis) of the $\mathrm{L} 6_{0}$ nanoprecipitates are fixed under magnetic fields.

Third, our work provides a feasible approach to overcome the trade-off between large magnetostriction and the small switching field of magnetostrictive materials. 
Although inducing uniaxial anisotropy by applying prestress or by magnetic annealing to redistribute the magnetic domains is effective in approaching the magnetostriction limit of a given material, it inevitably enlarges the switching field because the external field should first overcome the induced extra energy. The present work, which introduces mechanically harder tetragonal nanoprecipitates into a softer cubic matrix, overcomes this trade-off. On the one hand, the local tetragonal distortion of the matrix increases the spontaneous magnetostriction constant $\lambda_{001}$, i.e., the average tetragonality of the matrix. This, can be determined from a comparison of diffraction spots between the as-quenched and optimally aged samples, as shown at the bottom of Fig. 3d, e. On the other hand, although the locally distorted regions or the tetragonal nanoprecipitates may have stronger local MCA than the ideal cubic matrix, the random magnetic coupling at the nanoscale between them and the matrix results in the collapse of net MCA. According to Cullen et al. ${ }^{15},<001>\mathrm{Ga}-\mathrm{Ga}$ pairs can produce local uniaxial <001 $>$ anisotropy. The local regions with $<001>\mathrm{Ga}-\mathrm{Ga}$ pairs were suggested to form the $\mathrm{L6}_{0}$ (i.e., modified- $\mathrm{DO}_{3}$ ) structure ${ }^{35,36}$. Our atomicresolution STEM-HAADF Z-contrast image has directly shown that the $\mathrm{L} 6_{0}$ nanoprecipitates contain $<001>\mathrm{Ga}$ Ga pairs ${ }^{47}$. It can then be concluded that the $\mathrm{LG}_{0}$ nanoprecipitates have local uniaxial $<001>$ anisotropy, with the [001] easy axis parallel to one of the six equivalent $<001>$

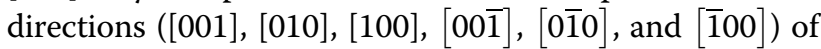
the BCC matrix due to simultaneous precipitation in local regions. The competition between the coherent crystalline anisotropy of the matrix and the anisotropy created by the fluctuations in the magnetization due to the local uniaxial $<001>$ anisotropy of the $\mathrm{L} 6_{0}$ nanoprecipitates then results in an instability of the $<001>$ uniformly magnetized state and manifests itself by the vanishing anisotropy. Following this, the locally distorted regions (strain nanodomains) may play a similar role as the $\mathrm{L}_{0}$ nanoprecipitates. However, how the magnetic couplings among the strain nanodomains, the $\mathrm{L} 6_{0}$ nanoprecipitates and the undistorted matrix quantitively affect the net anisotropy calls for further study. Since random short-range magnetic coupling is also known to improve magnetic susceptibility $^{14,63}$, the nanodispersive precipitates within the matrix can sensitively respond to low external fields, which accordingly reduces the switching field. With further development, this knowledge can be applied to obtain large sensitive magnetostriction in other nanostructured ferromagnets. We note that in Fe-Co alloys, quenching at the phase boundary between BCC and FCC can also create tetragonal nanoprecipitates, giving rise to larger and more sensitive magnetostriction when compared with the single BCC state $e^{3,64-66}$. Consequently, introducing dispersive tetragonal nanoprecipitates into a cubic matrix can be a universal approach to achieve large and sensitive magnetostriction. For engineering applications, heat treatment (short-term aging or quenching at specific temperatures) is a very simple approach when compared with single crystal growth and magnetic annealing approaches, which usually require special instruments and greater energy consumption. In addition, when compared with the approach of applying prestress, the present approach also has advantages in simplifying the design of magnetostriction-associated devices.

\section{Conclusions}

Our work provides a feasible approach to obtain large and sensitive magnetostriction in random polycrystalline ferromagnets by aging-induced nanodispersive precipitates. As shown in the proof-of-principle reference, $\mathrm{Fe}-\mathrm{Ga}$ alloys, short-term isothermal aging of the $\mathrm{BCC}$ precursor can effectively introduce FCT $\mathrm{L}_{0}$ nanoprecipitates. Manipulating the size and density of the $\mathrm{LG}_{0}$ nanoprecipitates significantly enhances the magnetostriction of the random polycrystals to be comparable to that for single crystal materials and effectively reduces the net magnetocrystalline anisotropy. The structural investigations reveal that the harder nanoprecipitates create local tetragonal distortion of the softer matrix (i.e., increasing the spontaneous tetragonal magnetostriction constant $\lambda_{001}$ ), resulting in magnetostriction enhancement. In situ magnetic domain structure observations and SANS measurements reveal that random magnetic coupling at the nanoscale effectively improves the magnetic susceptibility, hence giving rise to a 5 -fold enhancement in the figure of merit. These findings not only provide important insights toward understanding the microstructural origin of the extraordinary magnetostriction enhancement induced by soluting nonmagnetic Ga into Fe but also help to understand the heterogeneous magnetostriction mechanism. Aging-induced nanodispersive tetragonal precipitates within a cubic matrix can be a feasible approach to achieve large and sensitive magnetostriction in ferromagnetic materials.

\section{Acknowledgements \\ This work was supported by the National Natural Science Foundation of China (Grant Nos. 51871174, 52071256, and 51831006), the Young Talent Support Plans of XJTU and Shaanxi Province, the Fundamental Research Funds for Central Universities, the fund of the State Key Laboratory of Solidification Processing in NPU (Grant No. SKLSP202003), the Key Scientific and Technological Innovation Team of Shaanxi Province (2020TD-001), and the State Key Laboratory for Mechanical Behavior of Materials.}

\section{Author details}

${ }^{1}$ Frontier Institute of Science and Technology, State Key Laboratory for Mechanical Behavior of Materials, and MOE Key Laboratory for Nonequilibrium Synthesis and Modulation of Condensed Matter, Xi'an Jiaotong University, Xi'an 710049, China. ${ }^{2}$ School of Materials and Environmental Engineering, Hangzhou Dianzi University, Hangzhou 310012, China. ${ }^{3}$ Laboratory for Neutron Physics of Chinese Academy of Engineering Physics, Institute of Nuclear Physics and Chemistry, Mianyang 621999, China. ${ }^{4}$ CAS Key Laboratory of 
Magnetic Materials and Devices, Ningbo Institute of Materials Technology and Engineering, Chinese Academy of Sciences, Ningbo 315201, China. ${ }^{5}$ Ferroic Physics Group, National Institute for Materials Science, Tsukuba 305-0047 Ibaraki, Japan

\section{Conflict of interest}

The authors declare that they have no conflict of interest.

\section{Publisher's note}

Springer Nature remains neutral with regard to jurisdictional claims in published maps and institutional affiliations.

Supplementary information is available for this paper at https://doi.org/ 10.1038/s41427-020-00276-7.

Received: 8 January 2020 Revised: 1 November 2020 Accepted: 17 November 2020.

Published online: 15 January 2021

\section{References}

1. Chikazumi, S. Physics of Ferromagnetism (Oxford University Press, Oxford, UK 2005).

2. Clark, A. E. Magnetostrictive rare earth- $-\mathrm{Fe}_{2}$ compounds. Ferromagnetic Materials, 531-589 (North-Holland, Amsterdam, 1980).

3. Hunter, D. et al. Giant magnetostriction in annealed $\mathrm{CO}_{1-x} \mathrm{Fe} e_{x}$ thin-films. Nat. Commun. 2, 518 (2011).

4. Liu, J. H., Zhang, T. L., Wang, J. M. \& Jiang, C. B. Giant magnetostrictive materials and their applications. Mater. China 31, 1-12 (2012).

5. Chopra, H. D., Yang, X. D. \& Wilson, P. Magnetoelastic dependence of switching field in TbFe-FeCo giant magnetostrictive spring-magnet multilayers. J. Appl. Phys. 87, 5780-5782 (2000).

6. Atzmony, U. et al. Magnetic anisotropy and spin rotations in $\mathrm{Ho}_{x} \mathrm{~Tb}_{1-\mathrm{x}} \mathrm{Fe}_{2}$ cubic Laves compounds. Phys. Rev. Lett. 28, 244-247 (1972).

7. Yang, S. et al. Large magnetostriction from morphotropic phase boundary in ferromagnets. Phys. Rev. Lett. 104, 197201 (2010).

8. Bergstrom, Richard Jr et al. Morphotropic phase boundaries in ferromagnets: $\mathrm{Tb}_{1-x} \mathrm{Dy}_{x} \mathrm{Fe}_{2}$ alloys. Phys. Rev. Lett. 111, 017203 (2013).

9. Wun-Fogle, M., Restorff, J. B. \& Clark, A. E. Magnetostriction of stress-annealed Fe-Ga and Fe-Ga-Al alloys under compressive and tensile stress. J. Intell. Mater Syst. Struct. 17, 117-122 (2006).

10. Yoo, J.-H., Restorff, J. B., Wun-Fogle, M. \& Flatau, A. B. The effect of magnetic field annealing on single crystal iron gallium alloy. J. Appl. Phys. 103, 07 B325 (2008).

11. Zhang, C. S., Sun, G. A., Yan, M. \& Ma, T. Y. Effect of the induced anisotropy axis on altering domain alignment and magnetostriction of Terfenol-D. Appl. Phys. Lett. 104, 052409 (2014).

12. $\mathrm{Wu}, \mathrm{G}$. H. et al. $<111>$ oriented and twin-free single crystals of Terfenol-D grown by Czochralski method with cold crucible. Appl. Phys. Lett. 67, 2005-2007 (1995).

13. Rao, W. F. \& Khachaturyan, A. G. Superfunctionalities in nanodispersive precipitation-hardened alloys. Phys. Rev. Lett. 109, 115704 (2012).

14. Chudnovsky, E. M., Saslow, W. M. \& Serota, R. A. Ordering in ferromagnets with random anisotropy. Phys. Rev. B 33, 251-261 (1986).

15. Cullen, J., Zhao, P. \& Wuttig, M. Anisotropy of crystalline ferromagnets with defects. J. Appl. Phys. 101, 123922 (2007).

16. Guruswamy, S., Srisukhumbowornchai, N., Clark, A. E., Restorff, J. B. \& Wun-Fogle, M. Strong, ductile, and low-field-magnetostrictive alloys based on Fe-Ga. Scr. Mater. 43, 239-244 (2000).

17. Atulasimha, J. \& Flatau, A. B. A review of magnetostrictive iron-gallium alloys. Smart Mater. Struct. 20, 043001 (2011)

18. Petculescu, G., Wu, R. \& McQueeney, R. Magnetoelasticity of bcc Fe-Ga alloys. Handb. Magn. Mater. 20, 123-226 (2012).

19. Summers, E. M., Lograsso, T. A. \& Wun-Fogle, M. Magnetostriction of binary and ternary Fe-Ga alloys. J. Mater. Sci. 42, 9582-9594 (2007).

20. Jayaraman, T. V., Corson, R. P. \& Guruswamy, S. Ordering, magnetostriction, and elastic properties in Fe-27.5 at. \% Ga alloy single crystals. J. Appl. Phys. 102 053905 (2007)
21. Jin, C., Liu, J. H. \& Jiang, C. B. Magnetic domain characterization of high magnetic field treated FeGa single crystal. Mater. Res. Innov. 18, S4-597-S4-600 (2014).

22. Li, X. L., Bao, X. Q., Yu, X. \& Gao, X. X. Magnetostriction enhancement of $\mathrm{Fe}_{73} \mathrm{Ga}_{27}$ alloy by magnetic field annealing. Scr. Mater. 147, 64-68 (2018).

23. Ikeda, O., Kainuma, R., Ohnuma, I., Fukamichi, K. \& Ishida, K. Phase equilibria and stability of ordered b.c.c. phases in the Fe-rich portion of the Fe-Ga system. J. Alloy. Compd. 347, 198-205 (2002)

24. Srisukhumbowornchai, N. \& Guruswamy, S. Influence of ordering on the magnetostriction of Fe-27.5 at.\% Ga alloys. J. Appl. Phys. 92, 5371-5379 (2002).

25. Gou, J. M. et al. Tailoring magnetostriction sign of ferromagnetic composite by increasing magnetic field strength. Appl. Phys. Lett. 109, 082404 (2016).

26. Ma, T. Y. et al. Highly thermal-stable ferromagnetism by a natural composite. Nat. Comm. 8, 13937 (2017)

27. Palacheva, V. V. et al. Phase transitions as a tool for tailoring magnetostriction in intrinsic Fe-Ga composites. Acta Mater. 130, 229-239 (2017).

28. Fitchorov, T. I. et al. Thermally driven large magnetoresistance and magnetostriction in multifunctional magnetic FeGa-Tb alloys. Acta Mater. 73, 19-26 (2014).

29. Meng, C. Z., Wang, H., Wu, Y. Y., Liu, J. H. \& Jiang, C. B. Investigating enhanced mechanical properties in dual-phase Fe-Ga-Tb alloys. Sci. Rep. 6, 34258 (2016).

30. Dai, L., Cullen, J., Wuttig, M., Lograsso, T. \& Quandt, E. Magnetism, elasticity, and magnetostriction of FeCoGa alloys. J. Appl. Phys. 93, 8627-8629 (2003).

31. Xu, S. F. et al. Magnetostriction and electrical resistivity of $\mathrm{Mn}$ doped $\mathrm{Fe}_{81} \mathrm{Ga}_{19}$ alloys. J. Phys. D: Appl. Phys. 41, 015002 (2008)

32. Ren, $\mathrm{S}$. et al. Low-field-triggered large magnetostriction in iron-palladium strain glass alloys. Phys. Rev. Lett. 119, 125701 (2017).

33. Liu, J. J. et al. Magnetic transitions and magnetostrictive properties of $\mathrm{Tb}_{x} \mathrm{Dy_{1 }}$ $\left(\mathrm{Fe}_{0.8} \mathrm{Co}_{0.2}\right) 2(0.20 \leq \mathrm{x} \leq 0.40)$. Phys. Rev. B 75, 064429 (2007).

34. Xing, Q., Du, Y., McQueeney, R. J. \& Lograsso, T. A. Structural investigations of Fe-Ga alloys: phase relations and magnetostrictive behavior. Acta Mater. $\mathbf{5 6}$ 4536-4546 (2008).

35. Lograsso, T. A., Ross, A. R., Schlagel, D. L., Clark, A. E. \& Wun-Fogle, M. Structural transformations in quenched Fe-Ga alloys. J. Alloy. Compd. 350, 95-101 (2003).

36. He, Y. K. et al. Giant heterogeneous magnetostriction in Fe-Ga alloys: Effect of trace element doping. Acta Mater. 109, 177-186 (2016).

37. He, Y. K. et al. Interaction of trace rare-earth dopants and nanoheterogeneities induces giant magnetostriction in Fe-Ga alloys. Adv. Funct. Mater. 28, 1800858 (2018).

38. Jin, T. Y., Wang, H., Golovin, I. S. \& Jiang, C. B. Microstructure investigation on magnetostrictive $\mathrm{Fe}_{100-x} \mathrm{Ga}_{x}$ and $\left(\mathrm{Fe}_{100-x} \mathrm{Ga}_{x}\right)_{998} \mathrm{~Tb}_{02}$ alloys for $19 \leq \times \leq 29$. Intermetallics 115, 106628 (2019).

39. Lograsso, T. A. \& Summers, E. M. Detection and quantification of $\mathrm{DO}_{3}$ chemical order in Fe-Ga alloys using high resolution X-ray diffraction. Mater. Sci. Eng. A 416, 240-245 (2006)

40. Jen, S. U., Cheng, W. C. \& Chiang, F. L. Structural, magneto-mechanical, and damping properties of slowly-cooled polycrystalline $\mathrm{Fe}_{81} \mathrm{Ga}_{19}$ alloy. J. Alloy. Compd. 651, 544-550 (2015).

41. Rahman, N., Li, M. X., Ma, T. Y. \& Yan, M. Microstructural origin of the magnetostriction deterioration in slowly-cooled $\mathrm{Fe}_{81} \mathrm{Ga}_{19}$. J. Alloy. Compd. $\mathbf{7 8 6}$ 300-305 (2019).

42. Xing, Q. \& Lograsso, T. A. Effect of cooling rate on magnetoelasticity and shortrange order in Fe-Ga alloys. Scr. Mater. 65, 359-362 (2011).

43. Mohamed, A. K. et al. First- and second-order phase transitions in Fe-(17-19)at \%Ga alloys. Mater. Lett. 279, 128508 (2020).

44. Golovin, I. S., Balagurov, A. M., Bobrikov, I. A., Sumnikov, S. V. \& Mohamed, A. K. Cooling rate as a tool of tailoring structure of Fe-(9-33\%)Ga alloys. Intermetallics 114, 106610 (2019)

45. Balagurov, A. M., Samoylova, N. Y., Bobrikov, I. A., Sumnikov, S. V. \& Golovin, I. S. The first- and second-order isothermal phase transitions in $\mathrm{Fe}_{3} \mathrm{Ga}$-type compounds. Acta Cryst. B75, 1024-1033 (2019).

46. Khachaturyan, A. G. \& Viehland, D. Structurally heterogeneous model of extrinsic magnetostriction for Fe-Ga and similar magnetic alloys: part I. decomposition and confined displacive transformation. Metall. Mater. Trans. A 38, 2308-2316 (2007)

47. Gou, J. M., Yang, T. Z, Oiao, R. H., Liu, Y. \& Ma, T. Y. Format the ion mechanism of the tetragonal nanoprecipitates in Fe-Ga alloys that dominate material's large magnetostriction. Scr. Mater. 185, 129-133 (2020).

48. Wagner, W., Wiedenmann, A., Petry, W., Geibel, A. \& Gleiter, H. Magnetic microstructure of nanostructured $\mathrm{Fe}$, studied by small angle neutron scattering. J. Mater. Res. 6, 2305-2311 (1991). 
49. Laver, M. et al. Magnetostriction and magnetic heterogeneities in iron-gallium. Phys. Rev. Lett. 105, 027202 (2010).

50. Mudivarthi, C. Magnetic and structural characterization of Fe-Ga using Kerr microscopy and neutron scattering. (University of Maryland, College Park. PhD thesis, 2010).

51. Bischof, M. et al. The influence of spin-misalignment scattering on the SANS data evaluation of martensitic age-hardening steels. Acta Mater. 55, 2637-2646 (2007).

52. Michels, A. \& Weissmüller, J. Magnetic-field-dependent small angle neutron scattering on random anisotropy ferromagnets. Rep. Prog. Phys. 71, 066501 (2008).

53. Zhang, Y. N., Wang, H. \& Wu, R. Q. First-principles determination of the rhombohedral magnetostriction of $\mathrm{Fe}_{100-x} \mathrm{Al}_{x}$ and $\mathrm{Fe}_{00-x} \mathrm{Ga}_{x}$ alloys. Phys. Rev. $B$ 86, 224410 (2012).

54. Zhang, Y. N. \& Wu, R. Q. Mechanism of large magnetostriction of Galfenol. IEEE Trans. Magn. 47, 4044-4049 (2011).

55. Wang, $\mathrm{H}$. et al. Understanding strong magnetostriction in $\mathrm{Fe}_{100-x} \mathrm{Ga}_{\mathbf{x}}$ alloys. Sci. Rep. 3, 3521 (2013).

56. Ruffoni, M. P. et al. Direct measurement of intrinsic atomic scale magnetostriction. Phys. Rev. Lett. 101, 147202 (2008).

57. Bhattacharyya, S. et al. Nanodispersed $\mathrm{DO}_{3}$-phase nanostructures observed in magnetostrictive Fe-19\% Ga Galfenol alloys. Phys. Rev. B 77, 104107 (2008).
58. Rahman, N., Gou, J. M., Liu, X. L., Ma, T. Y. \& Yan, M. Enhanced magnetostriction of $\mathrm{Fe}_{81} \mathrm{Ga}_{19}$ by approaching an instable phase boundary. Scr. Mater. 146, 200-203 (2018).

59. Khachaturyan, A. G. \& Viehland, D. Structurally heterogeneous model of extrinsic magnetostriction for Fe-Ga and similar magnetic alloys: part II. giant magnetostriction and elastic softening. Metall. Mater. Trans. A 38, 2317-2328 (2007).

60. Ren, X. B. Strain glass and ferroic glass-Unusual properties from glassy nanodomains. Phys. Status Solidi B 251, 1982-1992 (2014).

61. Seiner, H., Kopecky, V., Landa, M. \& Heczko, O. Elasticity and magnetism of $\mathrm{Ni}_{2} \mathrm{MnG}$ a premartensitic tweed. Phys. Status Solidi B 251, 2097-2103 (2014).

62. Lloveras, P., Castán, T., Porta, M., Planes, A. \& Saxena, A. Influence of elastic anisotropy on structural nanoscale textures. Phys. Rev. Lett. 100, 165707 (2008).

63. Kronmüller, $P$. Theory of nucleation fields in inhomogeneous ferromagnets. Phys. Status Solid. B 144, 385-396 (1987).

64. Han, Y. J. et al. Tailoring the heterogeneous magnetostriction in Fe-Co alloys. J. Alloy. Compd. 699, 200-209 (2017).

65. Zhao, Y. L. et al. Magnetostriction and structure characterizations of $\mathrm{CO}_{70} \mathrm{Fe}_{30}$ alloy prepared by directional solidification. J. Magn. Magn. Mater. 451, 587-593 (2018).

66. Lisfi, A., Ren, T., Khachatuyan, A. \& Wuttig, M. Nano-magnetism of magnetostriction in $\mathrm{Fe}_{35} \mathrm{CO}_{65}$. Appl. Phys. Lett. 104, 092401 (2014). 